\title{
ORIGINAL
}

\section{LEGIBILIDAD DE LAS PÁGINAS WEB SOBRE SALUD DIRIGIDAS A PACIENTES Y LECTORES DE LA POBLACIÓN GENERAL}

Azucena Blanco Pérez (1) y Uxía Gutiérrez Couto (2)

(1) Consorcio Madroño de las Universidades de la Comunidad de Madrid y de la UNED

(2) Biblioteca del Complejo Hospitalario Arquitecto Maride. Ferrol. A Coruña.

\section{RESUMEN}

Fundamentos: Internet se está conformando como una de las fuentes de información sanitaria más importante. Al lado de esta popularidad surgen nuevas cuestiones tales como la calidad de la información o su capacidad de transmitir conocimiento. El objetivo es medir el grado de legibilidad de la información para pacientes accesible en Internet en lengua española.

Métodos: Se seleccionan 112 documentos acerca de 7 enfermedades crónicas. Recuperamos, de las estadísticas de legibilidad de Microsoft Word, los datos de número de sílabas y de oraciones en las 100 primeras palabras de cada documento. Para calcular la legibilidad aplicamos la adaptación de la fórmula de grado de Flesch para el idioma español realizada por Fernández Huerta.

Resultados: La media de legibilidad de los documentos es de $65,16( \pm 14,75)$, esto significa que se corresponde a un nivel estándar, es decir, un nivel de lectura de un niño de 13 años.

Conclusiones: Los índices de legibilidad que muestran los documentos analizados, aunque buenos, no son los óptimos para los pacientes y usuarios que buscan información de salud en Internet. La elaboración de documentos de información para los pacientes adaptados a su nivel de legibilidad es fundamental. Se necesitan de estudios que valoren las consecuencias que la baja alfabetización en salud como el uso incorrecto de los medicamentos, errores en la preparación para las exploraciones diagnósticas e incremento de los costes de los servicios de salud.

Palabras clave: Legibilidad. Educación para la salud. Internet.

\section{ABSTRACT}

\section{Readability of the Health Webpages for Patients and Readers among the General Population}

Background: Internet is becoming now becoming one of the major healthcare information sources. Aside from this popularity, new questions are now coming to bear, such as the quality of the information provided or its ability to convey knowledge. The purpose of this study is that of gauging the degree of readability of this information for patients which is accessible on the Internet in Spanish.

Methods: A total of 112 webpages dealing with seven (7) chronic diseases were selected. Based on the Microsoft Word readability statistics, we recovered the data of the number of syllables and sentences in the first 100 words in each document. For calculating readability, we used the adaptation of the Flesch degree formula for the Spanish language devised by Fernández Huerta.

Results: The mean readability of the documents is 65.16 14.75), thus corresponding to a standard level, in other words, a thirteen-year-old's reading level.

Conclusions: Although the readability scores found for the documents analyzed are good, they are not optimum for the users searching for health-related information on the Internet. It is essential that these informative documents be adapted to these users' readability level.. The need for studies evaluating the consequences entailed in the low degree of health-related literacy entails the incorrect use of medications, mistakes in preparing for diagnostic tests and an increase in the costs of healthcare services.

Keywords: Computer literacy. Patient education. Reading. Internet.

\section{INTRODUCCIÓN}

Correspondencia:

Azucena Blanco Pérez

Consorcio Madroño. Universidad Complutense de Madrid

Pabellón de gobierno

C/ Isaac Peral, s/n

28071 Madrid

Correo electrónico: ablanco@buc.ucm.es
El interés que despierta la información de salud en Internet es obvio. Algunas pruebas de este interés están en el aumento de portales de salud con información para usuarios, así como, las numerosas páginas web de sa- 
lud de distinto tipo: asociaciones de pacientes, organismos oficiales, páginas personales de pacientes y familiares afectados por determinadas dolencias. En la actualidad Internet facilita el acceso a cantidades incalculables de información. La presentación de la información relacionada con la salud en páginas web es cada vez más sofisticada, con avances manifiestos en la mejora de los contenidos, en la calidad y la fiabilidad de la información ${ }^{1-4}$ así como en la identificación de la autoría de los documentos. Pero al lado de estas mejoras se ponen sobre la mesa otros conceptos, como la «alfabetización en salud» (health literacy) y la legibilidad (readability) de los documentos de Internet, especialmente los dirigidos a los consumidores y población general.

El concepto de alfabetización en salud se utiliza por primera vez en el año 1974 en un informe de los Estados Unidos de Norteamérica acerca de la educación en salud como política social ${ }^{5}$. De las múltiples definiciones que surgen a partir de aquí nos quedamos con la que define la alfabetización como, «el grado por el cual los individuos tienen la capacidad de obtener, procesar y entender información básica de salud y los servicios que necesitan para tomar decisiones apropiadas en salud» ${ }^{6}$.

La legibilidad o la relativa dificultad de descifrar las palabras ${ }^{7}$ es uno de los factores requeridos para que la información escrita sea fácilmente comprensible para lectores de cualquier nivel. En Estados Unidos los problemas de alfabetización en salud emergen desde el momento en el que paciente solicita información sobre sus problemas de salud y asume una responsabilidad en la toma de decisiones sobre su estado de salud. En el mundo anglosajón el concepto está relacionado directamente con la educación en salud, la promoción de la salud y la prevención primaria.

Un análisis ${ }^{8}$ de la National Adult Literacy Survey de Estados Unidos de 1992 señala que alrededor de 90 millones de adultos americanos son analfabetos funcionales o marginalmente funcionales, teniendo dificultades para entender la información sobre los cuidados de salud que necesitan y reciben.

Un estudio realizado en dos hospitales públicos de Estados Unidos señala que el $31,5 \%$ de los pacientes de habla inglesa y el $61,7 \%$ de habla española presentan una inadecuada o marginal alfabetización funcional en salud ${ }^{9}$. Otro estudio presenta resultados semejantes en población anciana, es decir, el 33,9\% de los angloparlantes y el 53,9 de los pacientes de lengua española presentan una inadecuada o marginal alfabetización en salud $^{10}$. Los análisis del Departamento de Salud de Canadá ponen de manifiesto que el $53 \%$ de los adultos mayores de 65 años tienen un nivel 1 de alfabetización en salud ${ }^{11}$, lo que supone que son incapaces de reconocer en los folletos de medicamentos la dosis de medicina para suministrar a un niño. Una de las indicaciones de este estudio es la necesidad de analizar las consecuencias de esta baja alfabetización. Otro estudio revela que la capacidad de lectura y comprensión de los pacientes presentan grandes diferencias, pero, en general, es inferior al nivel de escolarización que poseen ${ }^{12}$. En este sentido, el Departamento de Salud de los Estados Unidos recomienda que los documentos para la educación de las personas estén adaptados al nivel de lectura del $6 .^{\circ}$ grado. Para poder apreciar mejor el nivel de legibilidad recomendado, hay que tener en cuenta que la equivalencia de los estudios del sistema educativo de los Estados Unidos de América con los correspondientes españoles ${ }^{13}$ equipara el $8 .^{\circ}$ grado de USA a $2 .^{\circ}$ grado de la ESO, es decir, un nivel de lectura de un niño de 13 años.

Otro de los aspectos que reflejan indirectamente los conceptos de legibilidad y alfabetización en salud que estamos analizando, se ha puesto de manifiesto en estudios americanos que revelan el coste económico de la escasa o baja alfabetización en salud existente en USA ${ }^{14}$. Por ejemplo, estancias hos- 
pitalarias más altas debido a la falta de comprensión de los planes de tratamiento y a la toma inadecuada de medicación que, según el estudio, suponen un gasto adicional de 73 billones de $\$$ en 1998. Además, se producen más visitas a los centros de salud y un mayor uso de los recursos hospitalarios especializados.

Existen más de 40 sistemas para medir la legibilidad de los textos ${ }^{15}$. Ninguno de ellos presenta una fórmula diseñada específicamente para textos médicos o relacionados con la salud 7 . Los modernos procesadores de textos ofrecen fórmulas para calcular la legibilidad, tales como: el nivel del grado de Flesch, la fórmula de la facilidad de la lectura de Flesch, el nivel de grado de Bormouth, el nivel de grado de Flesch-Kincaid. Otras fórmulas suponen el recuento manual, por ejemplo, la fórmula $\mathrm{SMOG}^{16}$ de legibilidad, y el Fry Readability Graph ${ }^{17}$. También está disponible software para realizar estas mediciones, por ejemplo, The Lexile Framework ${ }^{\circledR}$ y TextQuest. La literatura nos indica que si aplicamos las fórmulas mencionadas al mismo texto se producen resultados diferentes, se han llegado a encontrar variaciones de 1,3 a 3 niveles de grado entre diferentes programas ${ }^{18,19}$. El sistema que utilizan estas fórmulas es, en la mayoría de los casos, similar. Se seleccionan un número de frases de la primera parte del documento, el mismo número de frases de la mitad y la misma cantidad del final. Se contabilizan las sílabas de las palabras en cada grupo de frases, el número total de palabras contadas y se aplican las fórmulas oportunas. De todos los citados, el índice de Flesch-Kincaid se ha descrito como el más indicado para las instrucciones de autocuidados y para la información médica en general ${ }^{7}$. Flesch ideó esta fórmula para probar la legibilidad de los manuales de entrenamiento militar que, como las instrucciones de autocuidados y de información médica, han de ser comprensibles para adultos sometidos a tensión nerviosa y contienen, por necesidad, algún lenguaje técnico y términos poco corrientes. Kincaid ajustó la fórmula para obtener el ni- vel de lectura de un texto, adaptándose a un nivel de lectura de un instituto de los Estados Unidos.

El nivel de facilidad de lectura de Flesch (tabla 1) valora el texto en una escala de 100 puntos; cuanto más alto sea el resultado, más fácil será comprender el documento. Para la mayoría de los documentos estándar, el objetivo es un resultado comprendido entre 60 y 70 aproximadamente. La fórmula del nivel de facilidad de lectura de Flesch es: 206,835 - $(1,015 \times$ PLF $)-(84,6 \times$ PSP $)$. Donde, PLF es el promedio de longitud de frase (el número de palabras dividido por el número de frases) y PSP el promedio de sílabas por palabra (el número de sílabas dividido por el número de palabras). El nivel de Flesch-Kincaid valora el texto en un nivel de instituto de los EE.UU. Por ejemplo, un resultado de 8,0 indica que puede comprender el documento un estudiante de octavo. Para la mayoría de los documentos, el objetivo es un resultado comprendido entre $7,0 \mathrm{y}$ 8,0 aproximadamente.

Tabla 1

Grados en la Escala de Flesch (100 = muy fácil)

\begin{tabular}{|c|c|}
\hline Nivel & Dificultad de lectura \\
\hline $90-100$ & Muy fácil \\
\hline $80-90$ & Fácil \\
\hline $70-80$ & Un poco fácil \\
\hline $60-70$ & Estándar \\
\hline $50-60$ & Un poco difícil \\
\hline $30-50$ & Difícil \\
\hline $0-30$ & Muy difícil \\
\hline
\end{tabular}

Si activamos en el programa de tratamiento de texto de Microsoft Word 2000 herramientas/opciones, al finalizar la revisión ortográfica y gramatical de cada documento nos facilita diferentes indicadores: el grado de legibilidad en la escala Flesch, complejidad oracional, cómputos de palabras, de sílabas, de oraciones, etc.

Los índices automáticos de la escala Flesch que se obtienen no son válidos para 
el idioma español que utiliza frases y palabras más largas que el idioma inglés. Para las pruebas de legibilidad en otras lenguas ${ }^{20}$ deberán adaptarse las fórmulas de legibilidad, en este caso la fórmula Flesch, a las características específicas de cada idioma, en este caso al español.

El objetivo del presente estudio es conocer el grado de legibilidad de la información para usuarios y/o pacientes que se está elaborando escrita en español y disponible en Internet.

\section{MATERIAL Y MÉTODOS}

En nuestro caso, hemos seleccionado la adaptación de la fórmula Flesch para el idioma español que ha realizado Fernández Huerta $^{21}$. La fórmula de legibilidad es $=$ $206,84-0,60 \mathrm{P}-1,02 \mathrm{~F}$, debe aplicarse tomando varias páginas al azar de un texto, aconseja 30 para los libros y 5 para los artículos. En cada página o en cada muestra empezaremos siempre por el mismo párrafo y anotaremos el número total de sílabas por cada 100 palabras. El promedio del número de sílabas en los grupos de 100 palabras tomados se colocará en lugar de la P. En esos mismos párrafos se anota el conjunto de frases que constituyen cada cien palabras. El promedio de todas las muestras se coloca en lugar de la F. El resultado obtenido se compara con la tabla elaborada por Flesch (tabla 1).

Se transformaron los documentos, en formato htm o html en documentos word (.doc) para poder obtener los indicadores estadísticos. En este caso y, debido a que muchos de los documentos seleccionados no eran tan extensos para permitir la toma de muestras, se ha optado por analizar las 100 primeras palabras de cada documento. El recuento de las palabras se ha realizado con herramientas/contar palabras del programa de tratamiento de texto ya citado. Los indicadores de sílabas y de oraciones necesarios para la fórmula se han obtenido de los indicadores de cómputo que facilita la opción de estadísticas de legibilidad del programa. En una hoja de cálculo excel se configura la fórmula adaptada de Fernández Huerta para obtener los resultados de legibilidad de cada documento.

Los recursos de información para pacientes en Internet en español (tabla 2) se seleccionaron por diversos motivos: son portales cuyo fin es presentar información de salud para la población en general; contienen, en general, información sobre distintas enfermedades; son populares entre la población de habla hispana y sus documentos se localizan en buscadores generales como los que utiliza la población general para realizar búsquedas en la web. De estos recursos se eligieron 112 documentos que informaban acerca de 7 enfermedades crónicas: diabetes, asma, hipertensión arterial, cáncer de mama, cáncer de próstata, depresión y enfermedad pulmonar obstructiva crónica (EPOC). Los recursos web NOAH y Medline Plus se han desestimado por su cualidad de índices que nos reenvían a información elaborada en otros sitios web de salud para pacientes y, así, evitar la duplicidad de los documentos seleccionados.

Tabla 2

Recursos con información para pacientes en español seleccionados

\begin{tabular}{|c|c|}
\hline Nombre del recurso & $U R L$ \\
\hline Best Doctors & $\begin{array}{l}\text { http://www.bestdoctors.com/ } \\
\text { es/health_info.htm }\end{array}$ \\
\hline $\begin{array}{l}\text { Cancernet } \\
\text { (National Cancer Institute) }\end{array}$ & http://www.cancernet.gov \\
\hline Centro Médico Virtual & $\begin{array}{l}\text { http://www.centromedicovir- } \\
\text { tual.com }\end{array}$ \\
\hline Family doctor & $\begin{array}{l}\text { http://familydoctor.org/espa- } \\
\text { nol.html }\end{array}$ \\
\hline Gracias Doctor & http://www.graciasdoctor.com \\
\hline Onda salud & http://www.ondasalud.com \\
\hline Tu otro Médico & http://www.tuotromedico.com \\
\hline
\end{tabular}

Estas enfermedades han sido seleccionadas por ser las elegidas en otros estudios ${ }^{10,22}$ de similares características en Estados Uni- 
dos, por su condición de enfermedades crónicas que necesitan tratamientos constantes y buena comprensión de los cuidados necesarios y porque, además, afectan al sector de personas cuya capacidad de comprensión lectora se puede ver mermada en el transcurso de los años.

\section{RESULTADOS}

De los 112 documentos seleccionados (tabla 3) y valorados por la adaptación para el idioma español de la Escala de Flesch realizada por Fernández Huerta, 32 son de Centro Médico Virtual, 30 de Gracias Doctor, 28 de Tu Otro Médico, 12 de Family Doctor, 6 de Ondasalud, 2 de BestDoctors y otros 2 de Cancernet. El número de documentos valorados en cada sitio web se corresponden, no con la calidad, si no con la cantidad de información que contienen de cada una de las enfermedades que se habían seleccionado. Los documentos fueron escogidos aleatoriamente.

Los promedios de los resultados nos muestran que el nivel del grado de Flesch según la fórmula adaptada para el idioma español por Fernández Huerta es de 65,16 $( \pm 14,75)$, esto significa que se corresponde a un nivel estándar, apto para $7 .^{\circ}$ u $8 .^{\circ}$ grado, es decir, un nivel de lectura de un niño de 13 años.

Los documentos que mejor nivel de grado de Flesch adaptado presentan (tabla 4) en los datos de promedio son los de Gracias doctor 73,84. De los 12 documentos valorados, de la Web de Family Doctor, cuatro de ellos, están entre los 10 primeros documentos con mejor legibilidad; el promedio de legibilidad de los documentos de Family Doctor es 73,15 .

El 34,8\% (39) de los documentos presentan unos resultados entre 0 y 60 , es decir, un nivel de lectura de «un poco difícil» a «muy difícil», esto es, documentos que no alcanzan el estándar y, por lo tanto, para ser leí- dos, en el mejor de los casos, por estudiantes pre-universitarios.

\section{DISCUSIÓN}

Los resultados de nuestro estudio concuerdan, de forma general, con estudios previos sobre la legibilidad de la información para público no profesional, en los que se muestra que la legibilidad de la información disponible en Internet no es la adecuada, presentando los documentos un nivel de legibilidad excesivamente elevado. No obstante, la metodología y orientación de estos estudios difieren del nuestro. En el primer caso analizan documentos escritos exclusivamente en inglés y dirigidos a educación para los padres, además utilizan escalas de medición distintas como la Flesch Reading Ease, Flesch Kincaid, Fry y SMOG ${ }^{18}$. En otro caso, se analizan documentos para pacientes en inglés y español pero se utiliza la escala Fry de legibilidad ${ }^{22}$.

Establecer la legibilidad de un documento es una tarea complicada, fundamentalmente, por los diversos factores que intervienen en la comprensibilidad del texto, por ejemplo, la dificultad de las palabras utilizadas, el grado de simplicidad de las frases, las características y condiciones personales del lector, las características del texto y las imágenes y dibujos que ilustren el contenido.

Tener en cuenta las características del lector, en este caso pacientes y usuarios en general, a quienes va dirigido el texto que se escribe, es un aspecto fundamental en la elaboración de una información. Independientemente de la corrección gramatical, nuestro documento puede ser complejo o muy complejo en su estructura y léxico dificultando su comprensibilidad. Este factor es especialmente importante cuando se elabora información para presentarla en Internet, a la que cada día acceden más pacientes y población general de diferentes niveles de formación cultural $^{23}$. Los sistemas de recuento de las sílabas de las palabras y la longitud de las 
Resultados de legibilidad del Grado Flesch en la adaptación de Fernández Huerta para el idioma español

\begin{tabular}{|c|c|c|}
\hline Nombre del documento & Escala Huerta & Nombre del recurso \\
\hline Diabetes y su cuerpo. Como cuidar sus ojos y pies & 88,52 & Familydoctor \\
\hline Hipertensión arterial. & 88,34 & Tu otro médico \\
\hline ¿Qué es la hipertensión arterial? & 87,56 & Ondasalud \\
\hline Maternidad y diabetes & 86,54 & Gracias doctor \\
\hline Diabetes gestacional & 85,28 & Familydoctor \\
\hline Asma. Aprendiendo a controlar sus síntomas & 85,10 & Familydoctor \\
\hline El cáncer de seno y la menopausia & 84,56 & Gracias doctor \\
\hline Cancer de próstata & 84,08 & Tu otro médico \\
\hline Preeclampsia & 82,88 & Familydoctor \\
\hline Viviendo con... & 82,82 & Gracias doctor \\
\hline La depresión y la diabetes & 82,76 & Gracias doctor \\
\hline Cáncer de la próstata & 82,76 & Cancernet \\
\hline Diabetes: un futuro sin agujas & 82,16 & Gracias doctor \\
\hline Cáncer de mama & 82,1 & Tu otro médico \\
\hline Es importante guardar las mamografías & 81,38 & Gracias doctor \\
\hline Cáncer de próstata y su diagnóstico & 81,32 & Gracias doctor \\
\hline La importancia de la insulina & 80,96 & Gracias doctor \\
\hline ¿Qué es la depresión? & 80,96 & Ondasalud \\
\hline La diabetes y los problemas de la piel & 80,48 & Gracias doctor \\
\hline ¿Cómo se desarrolla la enfermedad? & 80,36 & Centro médico virtual \\
\hline ¿Qué es la bronquitis crónica? & 79,94 & Centro médico virtual \\
\hline ¿Qué es el cáncer de mama? & 79,52 & Centro médico virtual \\
\hline La fibra es esencial para los diabéticos & 79,34 & Gracias doctor \\
\hline Complicaciones de la diabetes & 78,32 & Tu otro médico \\
\hline El ejercicio disminuye los ataques de asma & 78,14 & Gracias doctor \\
\hline El cáncer de próstata & 78,14 & Gracias doctor \\
\hline Diabetes. Hacerse cargo de su diabetes & 77,48 & Familydoctor \\
\hline Asma & 77,06 & Gracias doctor \\
\hline Cáncer de mama: pasos para encontrar bultos temprano & 76,46 & Familydoctor \\
\hline Tipos de depresión & 76,16 & Centro médico virtual \\
\hline Depresión & 75,68 & Tu otro médico \\
\hline Pastilla para diabéticos & 74,96 & Gracias doctor \\
\hline ¿Qué es el cáncer? & 74,3 & Gracias doctor \\
\hline Tipos de diabetes & 74,12 & Centro médico virtual \\
\hline Prevención del cáncer de mama & 74,12 & Gracias doctor \\
\hline Pié diabético & 74,06 & Tu otro médico \\
\hline Hipertensión en el paciente adulto & 73,76 & Gracias doctor \\
\hline Complicaciones de la H.T.A. & 73,7 & Tu otro médico \\
\hline ¿Cuáles son las causas de la enfermedad? & 73,52 & Centro médico virtual \\
\hline ¿Cómo se manifiesta el cáncer de mama? & 73,4 & Centro médico virtual \\
\hline Se necesita más educación para los diabéticos acerca de su enfermedad & 73,16 & Gracias doctor \\
\hline ¿En qué consiste el enfisema? & 72,92 & Centro médico virtual \\
\hline Enfermedad Pulmonar Obstructiva Crónica (EPOC) & 71,72 & BestDoctors \\
\hline Medicación contra la diabetes afecta al hígado & 71,54 & Gracias doctor \\
\hline Presión sanguínea alta & 71,12 & Familydoctor \\
\hline Asma. Ocho preguntas para hacerse cuando no se mejora & 71,06 & Familydoctor \\
\hline Tratamiento de la hipertensión arterial & 70,88 & Centro médico virtual \\
\hline Cáncer de pecho: memoria y quimioterapia & 70,34 & Gracias doctor \\
\hline
\end{tabular}


Tabla 3 (continuación)

Resultados de legibilidad del Grado Flesch

en la adaptación de Fernández Huerta para el idioma español

\begin{tabular}{|c|c|c|}
\hline Nombre del documento & Escala Huerta & Nombre del recurso \\
\hline La alergia al polen & 70,16 & Gracias doctor \\
\hline Prevención de la diabetes & 69,92 & Gracias doctor \\
\hline La inyección de insulina & 68,66 & Tu otro médico \\
\hline Mamografía & 68,06 & Tu otro médico \\
\hline Autoanálisis de azúcar en la sangre & 67,88 & Tu otro médico \\
\hline Enfermedades pulmonares & 67,52 & Familydoctor \\
\hline Vitamina E y el corazón de los diabéticos & 67,34 & Gracias doctor \\
\hline Hipertensión arterial & 66,98 & Gracias doctor \\
\hline Hipertensión arterial & 66,98 & Gracias doctor \\
\hline ¿Por qué se producen las crisis asmáticas? & 66,56 & Centro médico virtual \\
\hline Asma. Tomando medicamentos de manera segura & 65,66 & Familydoctor \\
\hline Transplante celular: nueva esperanza para personas con diabetes tipo I & 65,54 & Gracias doctor \\
\hline Como tomarse uno la tensión & 64,7 & Tu otro médico \\
\hline ¿Qué es la diabetes? & 64,1 & Gracias doctor \\
\hline Tratamiento de la diabetes & 63,86 & Tu otro médico \\
\hline Asma bronquial & 63,38 & Tu otro médico \\
\hline Vigilando su presión arterial desde la casa & 62,9 & Familydoctor \\
\hline Alergia a animales caseros & 62,54 & Gracias doctor \\
\hline ¿Qué es la hipertensión arterial? & 62,36 & Centro médico virtual \\
\hline Signos y síntomas del cáncer de próstata & 61,94 & Centro médico virtual \\
\hline Autoexamen de las mamas & 61,58 & Tu otro médico \\
\hline Medicamentos para la hipertensión arterial & 61,52 & Tu otro médico \\
\hline Tipos de cáncer de mama & 61,1 & Centro médico virtual \\
\hline Complicaciones de la diabetes & 60,56 & Centro médico virtual \\
\hline Desarrollo y síntomas generales de la enfermedad & 60,14 & Centro médico virtual \\
\hline Tratamiento de la depresión & 59,72 & Centro médico virtual \\
\hline Manejo de los medicamentos para la H.T.A. & 59,72 & Tu otro médico \\
\hline Principales tipos de diabetes & 59,12 & Gracias doctor \\
\hline Enfermedad renal en la diabetes & 59,06 & Tu otro médico \\
\hline ¿Cuántos tipos de asma existen? & 58,34 & Centro médico virtual \\
\hline PSA. Antígeno específico de la próstata & 58,28 & Tu otro médico \\
\hline Hipoglucemia y la reacción a la insulina & 58,04 & Tu otro médico \\
\hline Bronquitis crónica, enfisema pulmonar, enfermedad obstructiva crónica & 57,5 & Centro médico virtual \\
\hline Cirugía para el cancer de mama & 57,08 & Tu otro médico \\
\hline Diabetes Mellitus & 56,6 & Tu otro médico \\
\hline Atención a los asmáticos & 54,92 & Gracias doctor \\
\hline ¿Cuándo, cómo y por qué aparece la diabetes? & 54,92 & Centro médico virtual \\
\hline ¿Qué es la enfermedad pulmonar obstructiva crónica? & 54,56 & Centro médico virtual \\
\hline Generalidades sobre la insulina & 54,26 & Tu otro médico \\
\hline ¿Qué es la depresión? & 53,96 & Centro médico virtual \\
\hline Retinopatía diabética & 53,48 & Tu otro médico \\
\hline Diabetes Mellitus & 51,92 & BestDoctors \\
\hline ¿Qué es el cáncer de próstata? & 51,74 & Centro médico virtual \\
\hline Lumpectomía & 51,2 & Tu otro médico \\
\hline ¿Qué es el asma bronquial? & 50,96 & Centro médico virtual \\
\hline ¿Qué es la diabetes? & 50,72 & Centro médico virtual \\
\hline Cáncer del seno (mama) & 50,12 & Cancernet \\
\hline ¿Qué es el cáncer de mama? Ondasalud.com & 50,12 & Ondasalud \\
\hline
\end{tabular}


Tabla 3 (continuación)

Resultados de legibilidad del Grado Flesch

en la adaptación de Fernández Huerta para el idioma español

\begin{tabular}{|c|c|c|}
\hline Nombre del documento & Escala Huerta & Nombre del recurso \\
\hline ¿Qué es el asma? & 49,76 & Ondasalud \\
\hline Grados y etapas del cáncer de próstata & 49,7 & Centro médico virtual \\
\hline Aparición y evolución del asma & 48,92 & Centro médico virtual \\
\hline Causas del cáncer de mama & 48,14 & Centro médico virtual \\
\hline Enfermedad pulmonar obstructiva crónica & 47,78 & Tu otro médico \\
\hline Diabetes y embarazo & 45,5 & Tu otro médico \\
\hline ¿Qué es la diabetes y tipos? & 45,32 & Ondasalud \\
\hline ¿Qué es el cáncer de próstata? & 45,32 & Ondasalud \\
\hline Prevención de la hipertensión arterial & 44,18 & Centro médico virtual \\
\hline Diabetes insípida & 43,82 & Familydoctor \\
\hline ¿Por qué se producen las depresiones? & 40,88 & Centro médico virtual \\
\hline ¿Qué causa la hipertensión arterial? & 36,32 & Centro médico virtual \\
\hline ¿Cuáles pueden ser las causas del cáncer de próstata? & 34,52 & Centro médico virtual \\
\hline Los factores de riesgo & 28,94 & Centro médico virtual \\
\hline Cirugía de la próstata & 19,34 & Tu otro médico \\
\hline Régimen dietético para la diabetes & 15,38 & Tu otro médico \\
\hline Complicaciones de la H.T.A. & 73,7 & Tu otro médico \\
\hline ¿Cuáles son las causas de la enfermedad? & 73,52 & Centro médico virtual \\
\hline ¿Cómo se manifiesta el cáncer de mama? & 73,4 & Centro médico virtual \\
\hline Se necesita más educación para los diabéticos acerca de su enfermedad & 73,16 & Gracias doctor \\
\hline ¿En qué consiste el enfisema? & 72,92 & Centro médico virtual \\
\hline Enfermedad Pulmonar Obstructiva Crónica (EPOC) & 71,72 & BestDoctors \\
\hline Medicación contra la diabetes afecta al hígado & 71,54 & Gracias doctor \\
\hline Presión sanguínea alta & 71,12 & Familydoctor \\
\hline Asma. Ocho preguntas para hacerse cuando no se mejora & 71,06 & Familydoctor \\
\hline Tratamiento de la hipertensión arterial & 70,88 & Centro médico virtual \\
\hline Cáncer de pecho: memoria y quimioterapia & 70,34 & Gracias doctor \\
\hline La alergia al polen & 70,16 & Gracias doctor \\
\hline Prevención de la diabetes & 69,92 & Gracias doctor \\
\hline La inyección de insulina & 68,66 & Tu otro médico \\
\hline Mamografía & 68,06 & Tu otro médico \\
\hline Autoanálisis de azúcar en la sangre & 67,88 & Tu otro médico \\
\hline Enfermedades pulmonares & 67,52 & Familydoctor \\
\hline Vitamina E y el corazón de los diabéticos & 67,34 & Gracias doctor \\
\hline Hipertensión arterial & 66,98 & Gracias doctor \\
\hline Hipertensión arterial & 66,98 & Gracias doctor \\
\hline ¿Por qué se producen las crisis asmáticas? & 66,56 & Centro médico virtual \\
\hline Asma. Tomando medicamentos de manera segura & 65,66 & Familydoctor \\
\hline Transplante celular: nueva esperanza para personas con diabetes tipo I & 65,54 & Gracias doctor \\
\hline Cómo tomarse uno la tensión & 64,7 & Tu otro médico \\
\hline ¿Qué es la diabetes? & 64,1 & Gracias doctor \\
\hline Tratamiento de la diabetes & 63,86 & Tu otro médico \\
\hline Asma bronquial & 63,38 & Tu otro médico \\
\hline Vigilando su presión arterial desde la casa & 62,9 & Familydoctor \\
\hline Alergia a animales caseros & 62,54 & Gracias doctor \\
\hline ¿Qué es la hipertensión arterial? & 62,36 & Centro médico virtual \\
\hline Signos y síntomas del cáncer de próstata & 61,94 & Centro médico virtual \\
\hline Autoexamen de las mamas & 61,58 & Tu otro médico \\
\hline
\end{tabular}


Tabla 3 (continuación)

Resultados de legibilidad del Grado Flesch

en la adaptación de Fernández Huerta para el idioma español

\begin{tabular}{|c|c|c|}
\hline Nombre del documento & Escala Huerta & Nombre del recurso \\
\hline Medicamentos para la hipertensión arterial & 61,52 & Tu otro médico \\
\hline Tipos de cáncer de mama & 61,1 & Centro médico virtual \\
\hline Complicaciones de la diabetes & 60,56 & Centro médico virtual \\
\hline Desarrollo y síntomas generales de la enfermedad & 60,14 & Centro médico virtual \\
\hline Tratamiento de la depresión & 59,72 & Centro médico virtual \\
\hline Manejo de los medicamentos para la H.T.A. & 59,72 & Tu otro médico \\
\hline Principales tipos de diabetes & 59,12 & Gracias doctor \\
\hline Enfermedad renal en la diabetes & 59,06 & Tu otro médico \\
\hline ¿Cuántos tipos de asma existen? & 58,34 & Centro médico virtual \\
\hline PSA. Antígeno específico de la próstata & 58,28 & Tu otro médico \\
\hline Hipoglucemia y la reacción a la insulina & 58,04 & Tu otro médico \\
\hline Bronquitis crónica, enfisema pulmonar, enfermedad obstructiva crónica & 57,5 & Centro médico virtual \\
\hline Cirugía para el cancer de mama & 57,08 & Tu otro médico \\
\hline Diabetes Mellitus & 56,6 & Tu otro médico \\
\hline Atención a los asmáticos & 54,92 & Gracias doctor \\
\hline ¿Cuándo, cómo y por qué aparece la diabetes? & 54,92 & Centro médico virtual \\
\hline ¿Qué es la enfermedad pulmonar obstructiva crónica? & 54,56 & Centro médico virtual \\
\hline Generalidades sobre la insulina & 54,26 & Tu otro médico \\
\hline ¿Qué es la depresión? & 53,96 & Centro médico virtual \\
\hline Retinopatía diabética & 53,48 & Tu otro médico \\
\hline Diabetes Mellitus & 51,92 & BestDoctors \\
\hline ¿Qué es el cáncer de próstata? & 51,74 & Centro médico virtual \\
\hline Lumpectomía & 51,2 & Tu otro médico \\
\hline ¿Qué es el asma bronquial? & 50,96 & Centro médico virtual \\
\hline ¿Qué es la diabetes? & 50,72 & Centro médico virtual \\
\hline Cáncer del seno (mama) & 50,12 & Cancernet \\
\hline ¿Qué es el cáncer de mama? Ondasalud.com & 50,12 & Ondasalud \\
\hline ¿Qué es el asma? & 49,76 & Ondasalud \\
\hline Grados y etapas del cáncer de próstata & 49,7 & Centro médico virtual \\
\hline Aparición y evolución del asma & 48,92 & Centro médico virtual \\
\hline Causas del cáncer de mama & 48,14 & Centro médico virtual \\
\hline Enfermedad pulmonar obstructiva crónica & 47,78 & Tu otro médico \\
\hline Diabetes y embarazo & 45,5 & Tu otro médico \\
\hline ¿Qué es la diabetes y tipos? & 45,32 & Ondasalud \\
\hline ¿Qué es el cáncer de próstata? & 45,32 & Ondasalud \\
\hline Prevención de la hipertensión arterial & 44,18 & Centro médico virtual \\
\hline Diabetes insípida & 43,82 & Familydoctor \\
\hline ¿Por qué se producen las depresiones? & 40,88 & Centro médico virtual \\
\hline ¿Qué causa la hipertensión arterial? & 36,32 & Centro médico virtual \\
\hline ¿Cuáles pueden ser las causas del cáncer de próstata? & 34,52 & Centro médico virtual \\
\hline Los factores de riesgo & 28,94 & Centro médico virtual \\
\hline Cirugía de la próstata & 19,34 & Tu otro médico \\
\hline Régimen dietético para la diabetes & 15,38 & Tu otro médico \\
\hline
\end{tabular}


Tabla 4

Resumen de resultados de legibilidad por sitio Web

\begin{tabular}{|ccc|}
\hline Sitio Web & N. ${ }^{\circ}$ de Documentos & Puntuación \\
\hline BestDoctors & 2 & $61,82(+/-14)$ \\
Cancernet & 2 & $66,44(+/-23)$ \\
Centro médico virtual & 32 & $58,67(+/-13)$ \\
Familydoctor & 12 & $73,15(+/-12)$ \\
Gracias doctor & 30 & $73,84(+/-7)$ \\
Ondasalud & 6 & $59,84(+/-19)$ \\
Tu otro médico & 28 & $61,13(+/-16)$ \\
\hline
\end{tabular}

frases, como hemos comentado, son muy similares; pero es importante destacar que estas fórmulas de legibilidad están valorando exclusivamente el texto de los documentos.

Ninguna de las fórmulas de legibilidad valora si los documentos presentan dibujos o información multimedia: vídeos, sonidos,... que permitan una mayor comprensión del documento. Sin duda, es uno de los aspectos que pueden incrementar la legibilidad y comprensibilidad de los documentos de información para pacientes en la web. El propio carácter multimedia de Internet, así como, la posibilidad de elaborar documentos de información no sólo con gráficos y dibujos, sino también, vídeos y juegos interactivos que puedan permitir al paciente comprobar que ha entendido bien el mensaje del documento, por ejemplo, que la dosis de medicación es la adecuada, paliará, en cierta medida, el bajo índice de legibilidad que algunos documentos presentan.

Los índices de legibilidad que muestran los documentos analizados, aunque buenos, no son los óptimos para los pacientes y usuarios que buscan información de salud en Internet. La información escrita debe ser un complemento a la comunicación entre médico y paciente. Es necesario trabajar para obtener documentos de información para los usuarios, adaptados a su nivel de legibilidad y grado de comprensión. La elaboración de esta información es de especial importancia, si se tienen en cuenta los numerosos problemas que puede generar el ac- ceso de los pacientes y consumidores a información obsoleta, con sesgos, etc.

El papel de los profesionales de la salud es fundamental para elaborar información para pacientes basada en la evidencia. Sería conveniente realizar estudios que valorasen las consecuencias que conlleva la baja alfabetización en salud: el uso incorrecto de los medicamentos y errores en la administración de los mismos; errores en la preparación de los pacientes para las pruebas diagnósticas y sus repercusiones económicas para el sistema sanitario, esto es el incremento en el uso de los servicios de salud. Habrá que añadir fórmulas de legibilidad en los procesadores de texto, validadas para el idioma español que faciliten el conocimiento del nivel de legibilidad de los documentos que se elaboran. También será necesario trabajar en nuevas fórmulas y sistemas que permitan medir la legibilidad de los documentos multimedia. Finalmente, si alguien se está preguntando por el resultado de legibilidad del texto de este artículo, es 64,96 con la fórmula Flesch adaptada al español de Fernández Huerta. Este indicador se corresponde con un grado de legibilidad estándar en la escala de grados de Flesch. Este artículo se ha elaborado para publicar en una revista científica especializada.

\section{AGRADECIMIENTOS}

Agradecemos al Dr. Rafael Bravo Toledo, médico de familia del área 10 de Madrid, su 
apoyo y asesoramiento en la elaboración de este trabajo, así como a los dos revisores anónimos por sus acertadas observaciones, que han contribuido a mejorar la redacción original de este estudio.

\section{BIBLIOGRAFÍA}

1. Wilson P. How to find the good and avoid the information on the Internet. BMJ 2002;324: 598-602.

2. Terry N. Regulation health information: a US perspective. BMJ 2002; 324: 602-6.

3. Winker MA. Normas para los sitios de información médica y de salud en Internet. Principios que rigen los sitios Web de la AMA. ACIMED 2001; 9(1): $109-22$.

4. Bergman MK. The deep Web: surfacing hidden value (julio 2000). http://www.brightplanet.com/deepcontent/tutorials/DeepWeb/deepwebwhitepaper.pdf; [citado octubre 2001]

5. Simonds SK. Health education as social policy. Health Educ Monogr 1974; 2:1-25.

6. Selden CR, Zorn M, Ratzan C, Parker RM. Current bibliographies in medicine 2000-1. Health Literacy; [citado 16 de nov. 2001] www.nlm.nih.gov/pubs/cbm/hliteracy.html.

7. Smith S. Probando la legibilidad de la información de salud; [citado 16 de nov. 2001] www.prenataled.com/story9.htm

8. U.S. Department of Education, Office of Educational Research and Improvement Adult literacy in America (consultado en noviembre 2001) http://www.nald.ca/fulltext/Report2/rep15-01.htm

9. Williams M, Parker R, Baker D, Parikh N, Pitkin $\mathrm{K}$, Coates W and Nurss J. Inadequate functional health literacy among patients at two public hospitals. JAMA 1995; 274 (21): 1677-82.

10. Gazmararian JA, Baker DW, Williams MV, Parker RM, Scott TL, Green DC, Fehrenbach SN, Ren J y Koplan JP. Health literacy among Medicare enrolees in managed care organization. JAMA 1999; 281 (6): 545-51

11. How Does Literacy Affect the Health of Canadians? [citado 21 de nov. 2001] www.hc-sc.gc.ca/hppb/ healthpromotiondevelopment/pube/literacyhealth/ literacy.htm

12. Health Information on the Internet. .[citado $18 \mathrm{de}$ nov. 2001] www.rand.org/publications/documents/interneteval

13. MEC. Información al ciudadano. Equivalencias de los estudios del sistema educativo de los Estados Unidos de América con los correspondientes españoles. [citado 10 de dic. 2001] www.mec.es/ inf/comoinfo/d-4-2-2-2.htm

14. Kilker K. Costs of low health literacy. .[citado 21 de nov. 2001] www.prenataled.com/healthlit/ hlt $2 \mathrm{k} / \mathrm{script} / \mathrm{index} . \mathrm{asp}$

15. Wieland $K$. Links to Readability Formulas and Information. [citado 18 de nov. 2001] www.gow. org/pages/About/resources11.html

16. University of Utah. Health Science Center. Guidelines for patient education. Written materials. An autor guide. The SMOG readability formula. Another readability option. .[citado 21 de nov. 2001] www.med.utah.edu/pated/authors/readability.html

17. Schrock K. FRY's Readability Graph: directions for use; [citado 3 de nov. 2001] school.discovery.com/schrockguide/fry/fry.html

18. D'Alesandro DM. The readability of pediatric patient education materials on the world wide web. Arch Pediatr Adolesc Med 2001; 155 (7): 807-12.

19. Mailloux SL. How reliable is computerized assessment of readability? Comput Nurs 1995; 13:221-5.

20. Rabin AT. Determining difficulty levels of text written in languages other than English. In: Zakaluk BL ,Samuels SJ, eds. Readability: Its past, present \& future. Newark DE: International Reading Association, 1988: 46-76

21. Fernández Huerta J. Medida sencillas de lecturabilidad. Consigna 1959; (214): 29-32

22. Berland GK, Elliot MN, Morales LS, Algazy JI, Kravitz RL, Broder MS. et al. Health information on the internet. Accesibility, quality and readability in english and spanish. JAMA 2001; 285(20): 2612-21.

23. Sarabia, AM. La accesibilidad en la red: no sólo de técnica vive el hombre. .[citado 28 de feb. 2002] www.timon.com/far/AMS032001.html. 